\title{
$202 \quad$ MONOCYTE SUBSET PHENOTYPE IN CORONARY ARTERY DISEASE AND THEIR ASSOCIATIONS WITH MARKERS OF INFLAMMATION AND FIBRINOLYSIS
}

L Tapp, E Shantsila, B Wrigley, S M Garcia, G Lip University of Birmingham Centre for Cardiovascular

doi:10.1136/heartjnl-2013-304019.202

Introduction The diverse roles of monocytes in the pathological mechanisms of atherogenesis and beneficial processes of myocardial repair are attributed to the existence of different subsets, distinguished by specific phenotypic and functional characteristics. However, scarce data are available on changes in phenotype and functional status of human monocyte subsets in coronary artery disease (CAD), especially when evaluated as three distinct subsets according to contemporary nomenclature.

Methods Monocyte surface expression of receptors implicated in inflammation and myocardial repair mechanisms was assessed by flow cytometry of venous blood in 53 patients with $\mathrm{CAD}$ and 50 age

Characterisitcs of monocyte subsets

\begin{tabular}{|c|c|c|c|c|c|c|c|c|c|}
\hline & \multicolumn{3}{|l|}{ Mon1 } & \multicolumn{3}{|l|}{ Mon2 } & \multicolumn{3}{|l|}{ Mon3 } \\
\hline & CAD & HC & p value & CAD & HC & p value & CAD & HC & p value \\
\hline Monocyte count, per $\mu \mathrm{l}$ & 469 [149] & $423[160]$ & 0.15 & 30 [18-48] & 29 [10-51] & 0.52 & 54 [39-79] & 49 [35-62] & 0.094 \\
\hline CD14, MFI & 1268 [342] & 1423 [312] & 0.02 & $1287[391]$ & $1281[495]$ & 0.95 & $141[44]$ & $158[36]$ & 0.036 \\
\hline CD16, MFI & $9[3]$ & $10[3]$ & 0.002 & $49[14]$ & $59[16]$ & 0.001 & $142[60]$ & $143[61]$ & 0.89 \\
\hline IL6r, MFI & 70 [16] & $62[20]$ & 0.025 & $64[14]$ & $55[20]$ & 0.015 & 35 [23] & $32[12]$ & 0.40 \\
\hline CXCR4, MFI & 17 [13] & $14[3.2]$ & 0.13 & 26 [17] & $21[8]$ & 0.069 & $9[6]$ & 6 [2] & 0.003 \\
\hline CD34, MFI & $2.2[0.3]$ & $2.0[0.2]$ & 0.007 & $2.1[0.5]$ & $1.8[0.3]$ & 0.002 & $2.0[0.4]$ & $1.6[0.3]$ & $<0.001$ \\
\hline VEGFR1, MFI & $6.3[1.6]$ & $6.8[1.8]$ & 0.28 & $13.3[4.7]$ & $15.2[6.4]$ & 0.13 & $4.2[1.5]$ & $4.0[1.8]$ & 0.55 \\
\hline CD163, MFI & $142[60]$ & $136[50]$ & 0.57 & 236 [90] & 222 [76] & 0.42 & 16 [12-22] & 14 [9-24] & 0.55 \\
\hline
\end{tabular}

Data presented as mean [standard deviation] or median [inter-quartile range]. MFI = median fluorescent intensity 
and sex-matched healthy controls (HC). Exclusion criteria comprised factors known to affect monocyte count. Monocyte subsets were defined as CD14++CD16-CCR2+(Mon1), CD14++CD16+CCR2 + (Mon2), and CD14+CD16++CCR2-(Mon3). Plasma levels of inflammatory cytokines (by cytometric bead array) and fibrinolytic factors (by enzyme-linked immunosorbent assay) were measured in patients with $\mathrm{CAD}$. Expression of surface markers was evaluated as median fluorescent intensity (MFI). The co-efficient of variability for these monocyte surface markers in our laboratory is $<5 \%$.

Results $\mathrm{CAD}$ was associated with reduced expression of CD14 on Mon1 $(p=0.02)$ and Mon3 $(p=0.036)$ and higher expression of IL6 receptor (IL6r) on Mon1 $(p=0.025)$ and Mon2 $(p=0.015)$, CXCR4 on Mon3 $(p=0.003)$, and CD34 on all subsets (all $p<0.007)$. Monocyte CD163 expression correlated negatively with plasma IL6 levels ( $p<0.01$ for all subsets). Plasma urokinase-type plasminogen activator levels correlated positively with VEGFR1 expression on Mon1 ( $r=0.51, p=0.003)$ and Mon2 $(r=0.44, p=0.012)$. VEGFR1 expression on Mon2 correlated positively with plasminogen activator inhibitor-1 (PAI-1) antigen levels $(r=0.47, p=0.006)$. IL6r expression by Mon2 correlated negatively with PAI- 1 antigen level $(r=-0.45$, $\mathrm{p}=0.005)$ and PAI- 1 activity $(\mathrm{r}=-0.47, \mathrm{p}=0.004)$.

Conclusions We describe significant novel changes in human monocyte subset phenotpye in $\mathrm{CAD}$, attributable to specific monocyte subsets, and a complex interplay with plasma cytokines and fibrinolytic factors. The pro-inflammatory IL6 pathway has been causally implicated in the pathogenesis of CAD, with IL6 remaining on effector cells being of key importance; this data suggests that Mon1 and Mon2 may represent such effector cells responsible for IL6r-mediated atherogenesis. Up-regualtion of CD34 expression by all monocyte subsets may represent a compensatory attempt to enhance angiogenesis in response to the persistent low-grade ischaemia present in $\mathrm{CAD}$. These data support the hypothesis for unique roles of the three human monocyte subsets in atherogenesis and $\mathrm{CAD}$ pathogenesis. Further research may allow for development of cell-specific therapeutic strategies for the modulation of atherosclerosis. The Mon2 subset and their relations with the IL6r pathway may represent a promising tar get. 